\title{
Rational Chebyshev Approximations for the Inverse of the Error Function
}

\author{
By J. M. Blair, C. A. Edwards and J. H. Johnson
}

\begin{abstract}
This report presents near-minimax rational approximations for the inverse of the error function inverf $x$, for $0 \leqslant x \leqslant 1-10^{-10000}$, with relative errors ranging down to $10^{-23}$. An asymptotic formula for the region $x \rightarrow 1$ is also given.
\end{abstract}

1. Introduction. The inverse error function inverf $x$ occurs in the solution of nonlinear heat and diffusion problems [1]. It provides exact solutions when the diffusion coefficient is concentration dependent, and may be used to solve certain moving interface problems. The percentage points of the normal distribution, which are important in statistical calculations, are expressible in terms of inverf $x$, and a common method of computing normally distributed random numbers [2], [3] requires efficient approximations.

The basic mathematical properties of the related function inverfc $x$ are discussed in [4] and [1], and 10S Chebyshev series expansions are given in [1]. [5] lists 3D rational approximations, and [6] contains $7 \mathrm{~S}$ rational minimax approximations to inverf $x$ and inverfc $x$. The most accurate set of approximations is given in [7], which contains Chebyshev series expansions accurate to at least $18 \mathrm{~S}$ for $0 \leqslant x \leqslant 1-10^{-300}$.

This report gives near-minimax rational approximations for inverf $x$ for $0 \leqslant x \leqslant$ $1-10^{-10000}$, with relative errors ranging down to $10^{-23}$. An asymptotic series is developed which gives at least twenty-five digits of accuracy over the remaining part of the range $1-10^{-10000} \leqslant x<1$. Tables $1-88$ computed by this method are included in the microfiche section of this issue. These tables provide the most efficient representations available, and the low order approximations should be useful in normal random number generators.

2. Functional Properties. The error function is defined for all real values of the argument $y$ by

$$
x=\operatorname{erf} y=2 \pi^{-1 / 2} \int_{0}^{y} e^{-t^{2}} d t
$$

and is an odd function of $y$. For $y \geqslant 0, x$ lies in the range $[0,1)$. The complementary error function is defined as

$$
\operatorname{erfc} y=1-\operatorname{erf} y \text {. }
$$

The inverse error function is defined by

Received December 15, 1975 ; revised March 16, 1976.

AMS (MOS) subject classifications (1970). Primary 65D20; Secondary 33A20, 41 A50.

Key words and phrases. Rational Chebyshev approximations, inverse error function, minimal Newton form. 


$$
y=\operatorname{inverf} x
$$

and the inverse error function complement by

$$
y=\operatorname{inverfc}(1-x) \text {. }
$$

inverf $x$ exists for $x$ in the range $-1<x<1$ and is an odd function of $x$, with a Maclaurin expansion of the form

$$
\text { inverf } x=\sum_{n=1}^{\infty} C_{n} x^{2 n-1}
$$

The first two hundred values of $C_{n}$ are listed in [7].

By inverting the standard asymptotic series

(1) erf $y \sim 1-\frac{\pi^{-1 / 2}}{y} e^{-y^{2}}\left[1+\sum_{m=1}^{\infty}(-1)^{m} \cdot \frac{1 \cdot 3 \cdot 5 \cdots(2 m-1)}{\left(2 y^{2}\right)^{m}}\right], \quad y \rightarrow \infty$,

we can derive an asymptotic expansion for inverf $x$ of the form

$$
\begin{aligned}
(\text { inverf } x)^{2} \sim & \eta-\frac{1}{2} \ln \eta+\eta^{-1}\left(\frac{1}{4} \ln \eta-\frac{1}{2}\right) \\
& +\eta^{-2}\left(\frac{1}{16} \ln ^{2} \eta-\frac{3}{8} \ln \eta+\frac{7}{8}\right) \\
& +\eta^{-3}\left(\frac{1}{48} \ln ^{3} \eta-\frac{7}{32} \ln ^{2} \eta+\frac{17}{16} \ln \eta-\frac{107}{48}\right) \\
& +\eta^{-4}\left(\frac{1}{128} \ln ^{4} \eta-\frac{23}{192} \ln ^{3} \eta+\frac{29}{32} \ln ^{2} \eta-\frac{31}{8} \ln \eta+\frac{1489}{192}\right) \\
& +\ldots, \quad x \rightarrow 1,
\end{aligned}
$$

where $\eta=-\ln \left[\pi^{1 / 2}(1-x)\right]$.

3. Generation of Approximations. Rational minimax approximations to inverf $x$ were computed in twenty-nine decimal arithmetic on a CDC 6600 using a version of the second algorithm of Remes due to Ralston [8]. The relative error of the approximations was levelled to three digits.

The approximation forms and intervals are

$$
\text { inverf } \begin{aligned}
x & \simeq x R_{l m}\left(x^{2}\right), & & 0 \leqslant x \leqslant 0.75, \\
& \simeq x R_{l m}\left(x^{2}\right), & & 0.75 \leqslant x \leqslant 0.9375, \\
& \simeq \xi^{-1} R_{l m}(\xi), & & 0.9375 \leqslant x \leqslant 1-10^{-100}, \\
& \simeq \xi^{-1} R_{l m}(\xi), & & 1-10^{-100} \leqslant x \leqslant 1-10^{-10000},
\end{aligned}
$$

where $R_{l m}(x)$ is a rational function of degree $l$ in the numerator and $m$ in the denominator, and where $\xi=[-\ln (1-x)]^{-1 / 2}$.

The auxiliary variable $\xi$ is necessary in the higher ranges to allow high accuracy approximations with rational functions of reasonable degree. The form of the asymptotic expansion (2) might suggest $\xi^{-1} R_{l m}\left(\xi^{2}\right)$ as a more natural approximating function. This form was checked, in addition to $\xi^{-1} R_{l m}(\xi)$ and $\xi^{-1} R_{l m}\left(\xi^{1 / 2}\right)$ for the highest 
range of $x$, and the latter found to be the most efficient. However, the improvement in accuracy is not enough to offset the cost of the additional square root evaluation.

For the range $0 \leqslant x \leqslant 0.9975$ the master routine computes inverf $x$ by solving the equation erf $y-x=0$ by the Newton-Raphson technique. For larger values of $x$, in the range $0.9975 \leqslant x \leqslant 1-e^{-625}$, we solve instead the equation erfc $y-(1-x)=0$. The computation of erf $y$ and erfc $y$ is based on the algorithm in [9], which was programmed in FORTRAN in 29S arithmetic on a CDC 6600. For $x>1-e^{-625}$ underflow occurs in evaluating $1-x$, and the equation is rewritten as $\xi(-\ln \operatorname{erfc} y)^{1 / 2}-1=$ 0 , where $\xi=[-\ln (1-x)]^{-1 / 2}$. Newton-Raphson iteration is again used, starting with $y=1 / \xi$, and the asymptotic formula (1) is used to compute erfe $y$. Because of the algorithms used, the computed values of inverf $x$ and inverfc $x$ are expected to be accurate to almost full-working precision.

The master routine was checked by comparing the results against the published formulae of Strecok [7]. The maximum relative differences for the ranges $[0,0.8]$, $[0.8,0.9975],\left[1-25 \times 10^{-4}, 1-5 \times 10^{-16}\right]$, and $\left[1-5 \times 10^{-16}, 1-10^{-300}\right]$ are $0.67 \times 10^{-24}, 0.13 \times 10^{-22}, 0.45 \times 10^{-22}$, and $0.39 \times 10^{-22}$, respectively, which are consistent with the magnitudes of the coefficients of the last terms retained by Strecok in his series expansions.

Additional checks consisted of a comparison of the results on either side of the transition points 0.9975 and $1-e^{-625}$, a comparison between the master routine and (2) at $x=1-10^{-10000}$, and differencing of the values generated by the master routine. The results indicate that the master routine is accurate to at least twentyseven digits.

4. Results. The details of the approximations are given in Tables $1-88$, in a format similar to that used in [10]. Tables 1-4 summarize the best approximations in the $L_{\infty}$ Walsh arrays of the function, and Tables 5-88 give the coefficients of selected approximations.

The precision is defined as

$$
-\log _{10} \max _{x}\left|\frac{f(x)-R_{l m}(x)}{f(x)}\right|,
$$

where $f(x)$ is the function being approximated, and the maximum is taken over the appropriate interval.

For the ranges $[0,0.75]$ and $[0.75,0.9375]$ the rational functions are ill-conditioned, both in the power polynomial and Chebyshev polynomial forms and lose up to three significant digits by cancellation. To eliminate the cancellation the numerator and denominator were subsequently converted to minimal Newton form (MNF) [11], and the resulting coefficients rounded off by an algorithm similar to that described in [10]. For each of the approximations in the tables the MNF has a particularly simple form, being a polynomial in $\left(x-x_{R}\right)$, where $x_{R}$ is the right-hand end of the approximation interval; and hence, the MNF is no more costly to evaluate than the power polynomial form. 
The approximations in Tables 5-88 were verified by comparing them with the master routine for 5000 pseudorandom values of the argument in each interval.

Atomic Energy of Canada Limited

Chalk River Nuclear Laboratories

Mathematics and Computation Branch

Chalk River, Ontario KoJ 1J0, Canada

Atomic Energy of Canada Limited

Chalk River Nuclear Laboratories

Mathematics and Computation Branch

Chalk River, Ontario KoJ 1J0, Canada

Statistics Canada

Business Service Methods Division

Coats Building

Tunney's Pasture

Ottawa, Ontario K1A 0T6, Canada

1. L. F. SHAMPINE, "Exact solutions for concentration dependent diffusion and the inverse complementary error function," J. Franklin Inst., v. 295, 1973, pp. 239-247.

2. M. E. MULLER, "An inverse method for the generation of random normal deviates on large-scale computers," MTAC, v. 12, 1958, pp. 167-174. MR 21 \#1690.

3. E. E. BATTISTE \& T. P. YEAGER, “GGNOR-generate pseudo-normal random numbers," IMSL Library 3 Reference Manual, v. 1, 1974

4. J. R. PHILIP, “The function inverfc $\theta$," Austral. J. Phys., v. 13, 1960, pp. 13-20. MR 22 \#9626.

5. C. HASTINGS, JR. (with J. T. HAYWARD \& J. P. WONG, JR.), Approximations for Digital Computers, Princeton Univ. Press, Princeton, N. J., 1955. MR 16, 963.

6. P. KINNUCAN \& H. KUKI, A Single Precision Inverse Error Function Subroutine, Computation Center, Univ. of Chicago, 1970.

7. A. J. STRECOK, "On the calculation of the inverse of the error function," Math. Comp., v. 22, 1968, pp. 144-158. MR 36 \#6119.

8. J. H. JOHNSON \& J. M. BLAIR, REMES 2-A FORTRAN Program to Calculate Rational Minimax Approximations to a Given Function, Report AECL-4210, Atomic Energy of Canada Limited, Chalk River, Ontario, 1973.

9. I. D. HILL \& S. A. JOYCE, "Algorithm 304. Normal curve integral [S15]," Comm. A.C.M., v. 10, 1967, pp. 374-375.

10. J. F. HART et al., Computer Approximations, Wiley, New York, 1968.

11. C. MESZTENYI \& C. WITZGALL, "Stable evaluation of polynomials," J. Res. Nat Bur. Standards Sect. B, v. 71 B, 1967, pp. 11-17. MR 35 \#3859. 